\title{
An experimental analysis of factors underlying neglect in line bisection
}

\author{
CHARLES M BUTTER, * VICTOR W MARK, $\dagger$ KENNETH M HEILMAN \\ From the Department of Neurology, College of Medicine, University of Florida, Gainesville, Florida USA
}

SUMMARY The finding that patients with neglect make larger errors when bisecting longer lines could be due to failure to disengage attention from a segment of the line on the ipsilesional side, or to a reduced ability to direct attention and/or action contralaterally. The findings are reported from a patient with left-sided neglect who set the midpoint further away from the right end of lines as their length increased, a finding consistent with the latter interpretation. His errors were significantly related to length and lateral extent of lines presented in left hemispace, but only to length of lines presented in right hemispace.

When patients with unilateral spatial neglect bisect horizontal lines, the size of their relative errors may increase as the length of the lines increases. ${ }^{1}$ This deficit may reflect failure to disengage attention from a segment of the line on the ipsilesional side. If this view is correct, the distance of the patient's setting from the attention-attracting end of the line should remain constant as the line length increases. Alternatively, neglect may be due to impairment of a system controlling attention and intention-action (including exploration) toward one side of space. We assume that the greater the value assigned by this system to a stimulus, the more likely the system is to attend and orient to it, so that it is perceived veridically. Assuming the system is distributed, ${ }^{2}$ following a cerebral lesion it would still be functioning, but in a degraded manner. Thus, it would still respond, although deficiently, to increasing demands placed on it, for example when bisecting lines of increasing length. By this view as line length increases, not only would the relative error of the neglect patient's bisection setting increase, but also the distance of the bisection setting from the ipsilesional end of the line would increase. We evaluated these two hypotheses by testing a patient

Present address: *Department of Psychology, University of Michigan, 1103 East Huron St, Ann Arbor, Michigan, USA. †Department of Neurology, School of Medicine, University of Maryland, Baltimore, Maryland, USA.

Received 5 January 1988 and in revised form 10 June 1988. Accepted 20 June 1988 with neglect in a bisection task with lines of varying length presented on both sides of space. To avoid confounding length of lines with their position in space, we also presented lines of constant length in different spatial positions.

\section{Subjects}

The patient was a 69 year old right-handed man who was transferred to the Gainesville Veteran's Administration Medical Center (GVAMC) in October, 1986 from another hospital following the acute onset of disorientation and left hemiparesis. He had a history of multiple strokes, chronic hypertension, diabetes and spondylitis. He regained full orientation by the third hospital day. He had a dense, left hemiparesis, including left lower facial weakness, and a left homonymous hemianopia. His deep tendon reflexes were more active on the left, but plantar responses were flexor. He recalled 2 of 3 objects in 5 minutes. Calculations with single digits and left-right discrimination were normal. His speech was slow, dysarthric and limited in melodic variation, but auditory comprehension, repetition, naming and writing were normal. He was impersistent when asked to keep his eyes closed. He showed left hemispatial neglect in line cancellation, line bisection, clock completion, writing, reading (he failed to read words on the left), figure copying, and in his drawings, which were grossly distorted.

Cranial computed tomography showed an acute, right temporo-occipital infarct, a small infarct in the right superior precentral gyrus and chronic, bilateral infarcts in the caudate nuclei. Electroencephalography showed slowing over the right hemisphere. Cerebrospinal fluid analysis was notable for mildly elevated protein level consistent with diabetes. He underwent the test described below 7 weeks after the onset of his most recent symptoms. 
Left

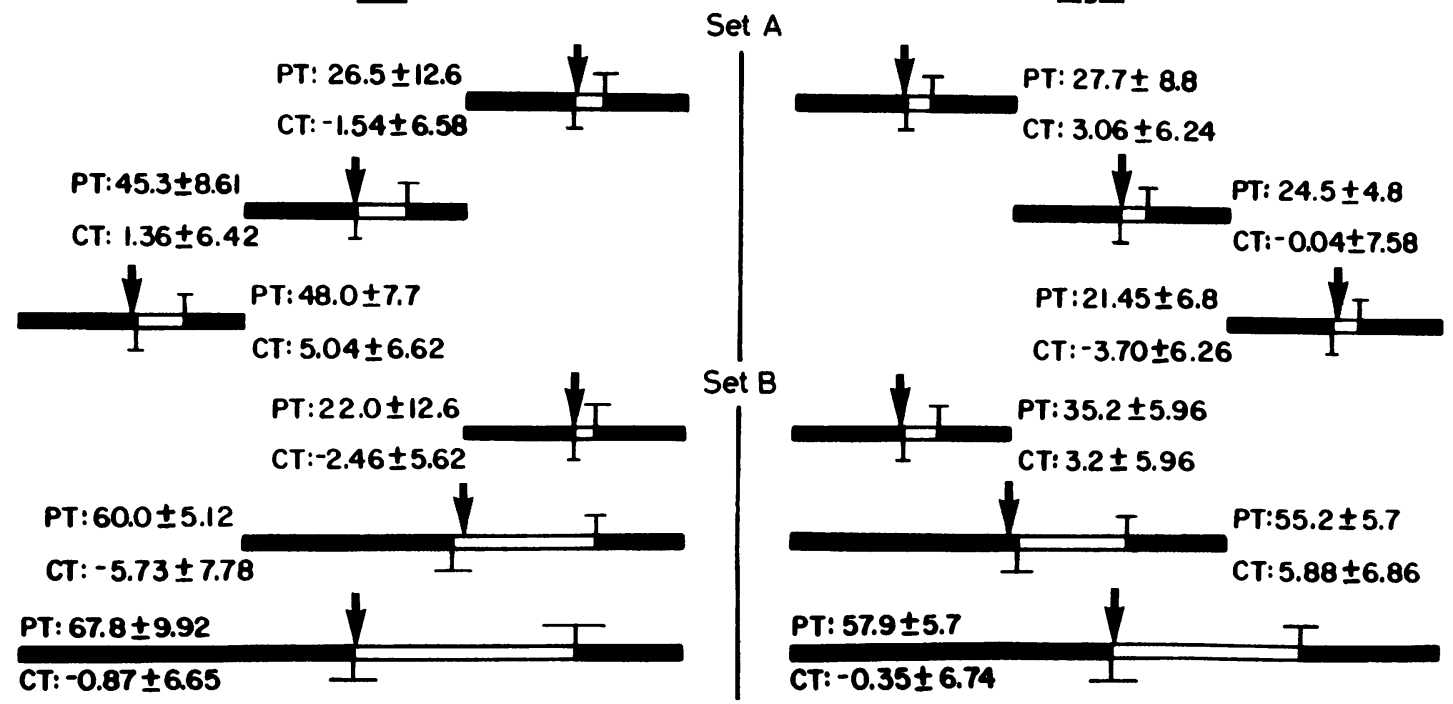

Fig Representations of the lines of constant length (set $A$ ) and variable length (set B) presented to the subjects on the left and right sides of space. The medial ends of the lines in set $A$ were $2.75 \mathrm{~cm}, 12.75 \mathrm{~cm}$ and $22.75 \mathrm{~cm}$ from the subject's midline axis. The medial ends of all the lines in set $B$ were $2.75 \mathrm{~cm}$ from the midline. The lateral ends of the 10 , 20 and $30 \mathrm{~cm}$ lines in set $B$ were located the same distances from the subjects' midline as were the lateral ends of the lines presented at the near, intermediate and far positions, respectively, in set $A$. Numbers refer to average relative errors (deviation of midpoint settings from true midpoint divided by distance of true midpoint from right end of line) and standard deviations of patient (PT) and control subjects (CT). Positive errors indicate deviations to the right of the true midpoint; negative errors indicate deviations to the left of the true midpoint. The short vertical bar above each line indicates the position of the average bisection setting of the patient; the cross-bar above each of these lines indicates the standard deviation of his settings. The short vertical bar below each line indicates the position of the control subjects' average bisection setting; the cross-bar above each of these lines indicates the standard deviation of their settings.

The control subjects were five, right-handed male inpatients at the Orthopaedic Service of the GVAMC. Their average age was 69 (range 61-78 years). They had no prior or current neurological disease; none showed neglect in standard tests.

\section{Methods}

Black lines, $0.3 \mathrm{~cm}$ thick, were presented on paper sheets (length $35.5 \mathrm{~cm}$, width $21.6 \mathrm{~cm}$ ) whose medial edges were aligned with the subject's body axis. Lines in set $A$, all $10 \mathrm{~cm}$ long, were presented at three distances from the midline of the body symmetrically on the two sides (see fig). Lines in set $\mathrm{B}, 10,20$ and $30 \mathrm{~cm}$ length, were presented symmetrically on the two sides (fig). The 12 lines were presented in a different random order in each of 12 successive blocks. The subjects were instructed to mark with a pencil the midpoint of the lines. They were permitted to move their head and eyes, but not their bodies while the lines were presented.

\section{Results and discussion}

Whereas the bisection settings of the control subjects did not differ significantly from the true midpoints of the lines in any of the 12 test conditions $(p>0 \cdot 1$ for all $t$ tests), those of the patient deviated significantly to the right $(p<0.001$ for all comparisons). Furthermore, the patient's relative errors to the right (his absolute errors divided by the distance from the midpoint to the right end of the line) increased significantly as the length of the lines in set $B$ increased $(F=101.038 ; d f$ $=2 / 66 ; p<0.0005$ ). This effect was due to increases in relative errors as line length changed from 10 to $20 \mathrm{~cm}$ and from 20 to $30 \mathrm{~cm}(p<0.025$ for both comparisons). This finding was not due to heightened attractiveness of the lines as they extended further into right hemispace, for his relative errors did not increase when the constant-length lines were displaced further into right hemispace (see fig). However, his relative errors did increase when constant length lines extended further into left hemispace $(p<0.05$, Tukey's hsd test), although not to the extent that they did when lines of increasing length were presented in left hemispace. This latter finding suggests the presence of a left 
hemispatial factor in the patient's performance, like that described by Heilman and Valenstein ${ }^{3}$ in neglect patients.

Although the patient's bisections of lines in set B showed marked errors, the distance of his settings from their right ends increased significantly as their lengths increased, irrespective of side of presentation $(p<0.05$, Tukey's hsd test), except when lines on the

Table Distances of settings from right ends of lines in set $B$ by patient (pt) and control subjects (ct)

\begin{tabular}{llrr}
\hline Line length (cm) & 10 & 20 & 30 \\
Distance of midpoint from right end of & & \multicolumn{1}{c}{} & \multicolumn{1}{c}{15} \\
$\quad$ line (cm) & 5 & 10 & 15 \\
Left pt & $3 \cdot 90$ & $4 \cdot 00$ & $4 \cdot 83$ \\
Side ct & $5 \cdot 12$ & $10 \cdot 57$ & $15 \cdot 13$ \\
Right pt & $3 \cdot 24$ & 4.48 & 6.23 \\
Side ct & $4 \cdot 84$ & 9.41 & 15.05 \\
\hline
\end{tabular}

left side increased from 10 to $20 \mathrm{~cm}$ (see table). These findings are at variance with the view that his neglect in line bisection was due to limitation of attention to a segment of the right side of the lines; they are consistent with the view that his increasingly severe neglect with longer lines was due to the degradation of a system directing attention/or action toward the neglected side.

\section{References}

1 Bisiach E, Bulgarelli C, Sterzi R, Vallar G. Line bisection and cognitive plasticity of unilateral neglect of space. Brain Cogn 1983;2:32-8.

2 Heilman KM, Bowers D, Coslett HB, Whelan H, Watson RT. Directional hypokinesia: prolonged reaction time for leftward movements in patients with right hemisphere lesions and neglect. Neurology 1985;35:855-60.

3 Heilman KM, Valenstein E. Mechanisms underlying hemispatial neglect. Ann Neurol 1979;5:166-70. 